\title{
Dynamics of Sorbitol at Elevated Pressure
}

\author{
Stella Hensel-Bielowka, ${ }^{\dagger}$ M. Paluch,,,$\dagger$ J. Ziolo, ${ }^{\dagger}$ and C. M. Roland ${ }^{*, \not}$ \\ Chemistry Division, Code 6120, Naval Research Laboratory, Washington, DC 20375-5342, and \\ Institute of Physics, Silesian University, Uniwersytecka 4, 40-007 Katowice, Poland
}

Received: July 1, 2002; In Final Form: August 29, 2002

\begin{abstract}
Dielectric relaxation measurements were carried out on sorbitol as a function of pressure at various temperatures. The almost linear dependence of the structural relaxation times on pressure yields values for the activation volume. In light of results for xylitol and glycerol, the activation volume is found to be an increasing function of molecular size. Because the pressure coefficients of the glass temperature for these polyalcohols are all equal, their fragilities should parallel their respective activation volumes. This expectation is borne out by experimental measurements at ambient pressure. Analysis of the volume dependence of the relaxation times reveals that temperature, rather than density, dominates the structural relaxation of sorbitol. At frequencies higher than the structural relaxation, a secondary process is observed. The weaker pressure sensitivity of the latter effects better resolution of the two peaks at high pressures. The relaxation time for the secondary process is consistent with a calculation from the coupling model based on identification of the secondary process as the precursor to the highly cooperative structural relaxation.
\end{abstract}

\section{Introduction}

Structural relaxation of supercooled liquids continues to evoke great interest due to the diverse behaviors observed, as well as the fundamental significance of the phenomenon. Some recent progress has been achieved by extending experimental studies to the effect of hydrostatic pressure on the relaxation dynamics. Examples of the utility of pressure as a variable include discovery of unanticipated structure-property relationships, ${ }^{1,2}$ resolution of overlapping spectral dispersions, ${ }^{3,4}$ assessment of the relative contributions of free volume and thermal energy to the dynamics, ${ }^{5-9}$ and providing new insight into the development of intermolecular cooperativity near the glass temperature. $^{10}$

Among the myriad glasses, the added complexity of associated liquids makes them especially interesting. Sorbitol is a six carbon polyalcohol characterized by extensive hydrogen bonding. Its large dielectric strength has caused it to be the subject of numerous investigations using dielectric spectroscopy. ${ }^{11-19}$ Sorbitol also has an experimentally convenient glass temperature, $T_{\mathrm{g}} \approx 264^{11}$ to $273 \mathrm{~K},{ }^{20}$ and is very "fragile", $m \equiv \mathrm{d} \log$ $\left(\tau_{\alpha}\right) /\left.\mathrm{d}\left(T_{\mathrm{g}} / T\right)\right|_{T=T_{\mathrm{g}}}=128 .{ }^{16}$ Consistent with this large $m$, the structural relaxation peak in the dielectric loss is quite broad, fwhm $>3$ decades. ${ }^{11}$ At ambient pressure, the structural relaxation times follow the Vogel equation

$$
\tau_{\alpha}(T)=\tau_{\infty} \exp \left(\frac{B}{T-T_{\infty}}\right)
$$

for $300 \mathrm{~K}>T>T_{\mathrm{g}}$ with deviation to a different Vogel behavior observed at higher temperatures. ${ }^{17}$ Sorbitol has a prominent secondary peak in the dielectric loss spectrum. From the glassy state up to temperatures as high as $293 \mathrm{~K}$, this $\beta$-relaxation exhibits an Arrhenius temperature dependence with an activation equal to $68 \pm 7 \mathrm{~kJ} / \mathrm{mol}^{12,16}$

* To whom correspondence should be addressed.

Silesian University.

$\doteqdot$ Naval Research Laboratory.
In this paper, we report dielectric spectroscopy data for sorbitol under elevated pressure. Naoki and Katahira ${ }^{11}$ previously studied the primary structural relaxation, using dielectric measurements covering 3 decades of frequency at $78.5 \mathrm{MPa}$. They interpreted their results in terms of the competing effects of thermal energy, free volume, and the pressure-dependent extent of hydrogen bonding. We extend this work with measurements over 9 decades of frequency at a series of pressures up through $400 \mathrm{MPa}$ at each of three temperatures. The response to pressure of the structural relaxation, as well as the secondary relaxation process in sorbitol, is investigated. In addition to providing new insights into the behavior of sorbitol, the results are compared to literature data for homologous polyalcohols, yielding interesting structure-property information.

\section{Experimental Section}

The sorbitol (synonyms, D-sorbitol or glucitol) was obtained from Aldrich and used as received. The complex dielectric permittivity, $\epsilon^{*}=\epsilon^{\prime}-i \epsilon^{\prime \prime}$, was measured in the frequency range from $10^{-2}$ up to $10^{7} \mathrm{~Hz}$ using an Novocontrol Alpha analyzer. The capacitor consists of two parallel stainless steel plates separated by a quartz spacer and has a nominal geometric capacitance of about $10 \mathrm{pF}$. The electrodes are mounted inside a cylindrical capsule. After filling with the test liquid, the cell, sealed and mounted inside a Teflon ring, is placed in a highpressure chamber. Pressure is exerted via silicone fluid using a piston in contact with a hydraulic press. Deformation of the Teflon membrane covering the top of the cylindrical capsule pressurizes the sample. The pressure was measured by a Nova Swiss tensometric pressure meter with a resolution of $0.1 \mathrm{MPa}$. The temperature was controlled to within $0.1 \mathrm{~K}$ by means of liquid flow from a thermostatic bath.

\section{Results}

Figure 1 shows a representative dispersion in the dielectric loss, $\epsilon^{\prime \prime}(\omega)$, for sorbitol measured at elevated pressure. The dc 


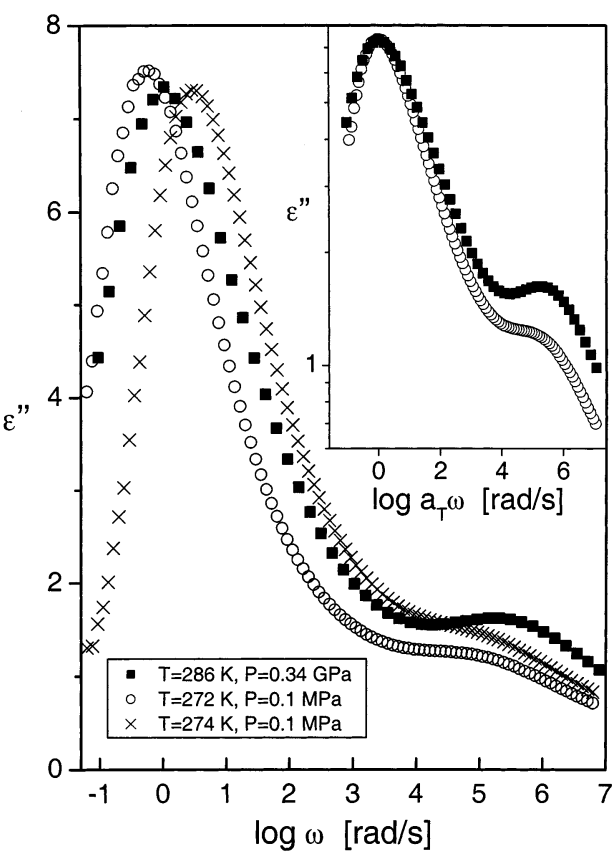

Figure 1. The dielectric loss of sorbitol (ם) at elevated pressure (340 $\mathrm{MPa}$ ) at $286.4 \mathrm{~K}$ and at atmospheric pressure at (O) 272 and $(\times) 274$ $\mathrm{K} \times$. The inset shows the superposition obtained by shifting the $272 \mathrm{~K}$ spectrum by 0.8 decades. The conductivity contribution, assumed proportional to $1 / \omega$, has been subtracted from the spectra.

conductivity at low frequencies has been subtracted from the spectrum by assuming an inverse proportionality to log frequency. The respective temperatures of the three spectra were chosen such that the $\tau_{\alpha}$ (defined as the inverse of the circular frequency, $\omega$, of the maximum in the dielectric loss) were essentially the same; thus, the higher pressure spectrum corresponds to a higher temperature. The secondary relaxation is in evidence toward higher frequencies, with an increased relative prominence in comparison to the spectra at ambient pressure. This is illustrated in the inset, showing the superposition of the structural relaxation peaks measured at ambient and high pressure. The latter corresponds to higher temperature to match the $\tau_{\alpha}$. Consequently, the prominence of the $\beta$-process is enhanced because above $T_{\mathrm{g}}$ the dielectric strength, $\Delta \epsilon$, of the primary relaxation for sorbitol decreases with temperature while that of the secondary relaxation increases. ${ }^{15}$ In addition, $\Delta \epsilon$ of the primary relaxation decreases with pressure. ${ }^{11}$

The greater resolution of the two peaks is also a consequence of their differing response to pressure. As shown in Figure 2, the structural relaxation times vary more strongly with pressure. For the lower two temperatures in Figure 2, the data vary roughly in a linear fashion with pressure over the measured range; thus, a simple, volume-activated model suffices, ${ }^{21}$

$$
\tau_{\alpha}=\tau_{\infty} \exp \left(\frac{P \Delta V}{R T}\right)
$$

where $R$ is the gas constant and $\Delta V$ is an activation volume. The best-fit values of $\tau_{\infty}$ and $\Delta V$ are listed in Table 1 . These activation volumes are about $75 \%$ of the molar volume of sorbitol.

For the data at the highest temperature in Figure 2, nonlinearity is evident in the $\tau_{\alpha}(P)$ behavior; accordingly, for $T=$ $303 \mathrm{~K}$, we use the expression ${ }^{22,23}$

$$
\tau_{\alpha}=\tau_{\infty} \exp \left(\frac{D P}{P_{0}-P}\right)
$$

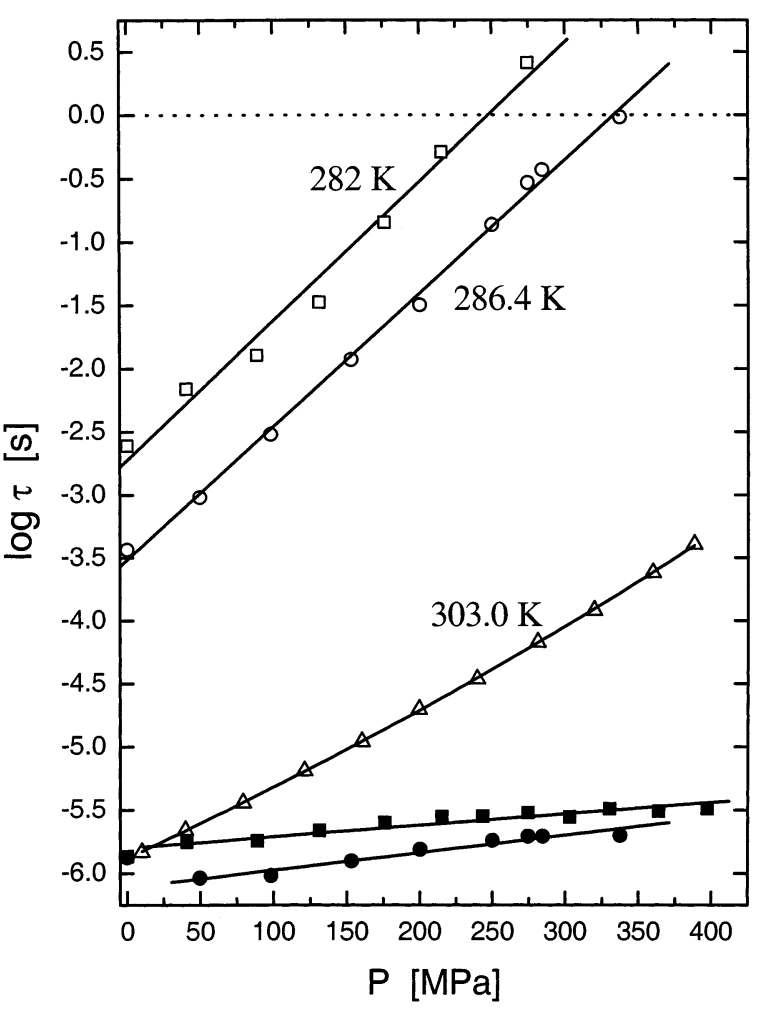

Figure 2. The structural (open symbols) and secondary (filled symbols) relaxation times for sorbitol as a function of pressure: temperature $=$ ( $\square$ ) 282.0; (O) 286.4, and $(\triangle) 303.0 \mathrm{~K}$. The solid curves represent straight-line fits to the data, except for $\tau_{\alpha}$ at $303 \mathrm{~K}$, which were fit to eq 3 . Note these data were obtained at frequencies slower than the crossover frequency $\left(\sim 10^{6} \mathrm{~Hz}\right)$, associated with a change in dynamics and merging of the primary and secondary processes. ${ }^{14}$

TABLE 1: Results for Sorbitol

\begin{tabular}{lllc}
\hline$T(\mathrm{~K})$ & process & \multicolumn{1}{l}{$\log \tau_{\infty}(\mathrm{s})$} & $\Delta V(\mathrm{ml} / \mathrm{mol})$ \\
\hline 282 & structural & $-2.72 \pm 0.1$ & $59.4 \pm 3.4$ \\
& secondary & $-5.80 \pm 0.02$ & $4.6 \pm 0.5$ \\
286.4 & structural & $-3.52 \pm 0.05$ & $58.2 \pm 1.2$ \\
& secondary & $-6.01 \pm 0.05$ & $6.5 \pm 1.2$ \\
303 & structural & $-5.95 \pm 0.02$ & $33.1 \pm 0.7^{a}$
\end{tabular}

${ }^{a}$ At $P<100 \mathrm{MPa}$.

in which $D$ and $P_{0}$ are material constants. We obtain $D=30.9$ and $P_{0}=2490 \mathrm{MPa}$ with the values of $\tau_{\infty}$ and $\Delta V=(R T \partial \ln$ $\left.\tau_{\alpha} /\left.\partial P\right|_{T}\right)$ at lower pressures given in Table 1 . Note that eq 3 could be used equally well to describe the structural relaxation times measured at the two lower temperatures. Indeed, the fact that $P_{0}$ for $303 \mathrm{~K}$ is significantly larger than the applied pressures in Figure 1 indicates that the deviation from proportionality between $\log \tau_{\alpha}$ and $P$ is small. Certainly, the use of different equations is not meant to imply that any change in behavior occurs over the temperature range from 282 to $303 \mathrm{~K}$.

We can define a dynamic glass transition as the temperature at which the relaxation time equals $1 \mathrm{~s}$. This avoids the extrapolation necessary to use the more customary $T_{\mathrm{g}}\left(\tau_{\alpha}=100 \mathrm{~s}\right)$. At ambient pressure, $T_{\mathrm{g}}=272.4 \mathrm{~K}$, which is close to the value obtained by thermal analysis. ${ }^{20}$ As seen in Figure $2, T_{\mathrm{g}}$ at elevated pressures can be obtained directly from measurements at the two lower temperatures. The variation with pressure is linear, yielding $\mathrm{d} T_{\mathrm{g}} / \mathrm{d} P=0.040 \pm 0.003 \mathrm{~K} / \mathrm{MPa}$. Extrapolation of the fit to eq 3 for the data obtained at $T=303 \mathrm{~K}$ is consistent with this value of the pressure coefficient, indicating that linearity may extend up through $730 \mathrm{MPa}$.

The relaxation times for the secondary relaxation, $\tau_{\beta}$, are also displayed in Figure 2. The data suggest a weak pressure 
dependence. We fit all of the secondary relaxation times for sorbitol to eq 2 , obtaining an activation volume, $5 \pm 1 \mathrm{~mL} /$ mol, that is an order of magnitude smaller than $\Delta V$ for structural relaxation (Table 1). Thus, the weaker sensitivity to pressure of the secondary relaxation results in an increasing separation of the $\alpha$ - and $\beta$-processes with increasing pressure.

\section{Discussion}

The greater resolution of sorbitol's primary and secondary relaxations is due in part to the enhanced prominence of the latter at higher pressures. This enhancement would seem to argue against an interpretation of the $\beta$-process as being due to islands of mobility within a highly constrained supercooled liquid matrix. ${ }^{24,25}$ Otherwise, one would expect the increasing density associated with higher pressure to reduce this mobility and thus suppress the $\beta$-process. Also contributing to the greater separation of the two processes at any fixed temperature is the much greater sensitivity of the $\alpha$-relaxation times to pressure, in comparison to the $P$ dependence of $\tau_{\beta}$. In terms of the activation volumes, there is an order of magnitude difference.

However, although the activation volume for the secondary process is small relative to $\Delta V$ for structural relaxation, the former is not zero (Table 1). This finite pressure sensitivity is consistent with the $\beta$-process having an intermolecular origin; that is, it is a Johari-Goldstein process. ${ }^{26}$ This is unsurprising, in light of the structure of sorbitol (namely, the absence of pendant chemical groups). This can be compared to the secondary peak in the dielectric spectrum of $1,1^{\prime}$-bis ( $p$-methoxyphenyl) cyclohexane, which is known from NMR measurements to arise from flipping motion of the methoxyphenyl rings. ${ }^{27}$ It was also recently found that the secondary relaxation times measured dielectrically are invariant to pressure. ${ }^{28}$ The implication is that this local motion, not involving the entire molecule, may not be a Johari-Goldstein process. On the other hand, a Johari-Goldstein secondary peak in chlorobenzene/ decalin mixtures did exhibit a frequency shift with change in pressure. $^{29}$

For Johari-Goldstein secondary relaxations, a quantitative relationship between $\tau_{\alpha}$ and $\tau_{\beta}$ has been proposed ${ }^{30,31}$

$$
\tau_{\beta}=t_{\mathrm{c}}{ }^{1-\beta_{\mathrm{K}}} \tau_{\alpha}{ }^{\beta_{\mathrm{K}}}
$$

where $t_{\mathrm{c}}(\sim 2 \mathrm{ps})$ is a temperature-independent constant and $\beta_{\mathrm{K}}$ is the Kohlrausch fractional exponent characterizing the deviation of the dielectric loss peak from exponential decay ${ }^{32,33}$

$$
\epsilon^{\prime \prime}(\omega)=\Delta \epsilon \int_{0}^{\infty} \mathrm{d} t\left[\frac{-\mathrm{d}}{\mathrm{d} t} \exp -\left(t / \tau_{\mathrm{K}}\right)^{\beta_{\mathrm{K}}}\right] \sin (\omega t)
$$

(The Kohlrausch relaxation time, $\tau_{\mathrm{K}}$, in eq 5 is somewhat less than the reciprocal of the peak frequency.) Equation 4 relating the two time constants is derived from a treatment of the JohariGoldstein process as transpiring at times sufficient for many units to be attempting to relax, whereby some degree of cooperativity has become necessary. This secondary process thus serves as the precursor to the fully cooperative $\alpha$-relaxation, occurring at longer times when all molecules attempt relaxation but are preempted by cooperativity. The separation of the two processes is then related, per eq 4 , by the magnitude of $\beta_{\mathrm{K}}{ }^{30}$

In Figure 3, we display the measured dielectric loss for sorbitol at $250 \mathrm{MPa}$ and $286.4 \mathrm{~K}$ after subtraction of the dc conductivity. Because the secondary peak contributes to the dielectric loss on the high-frequency side of the structural relaxation peak, in fitting eq 5 , we emphasize the low-frequency side of the primary peak. The obtained fit, shown in Figure 3,

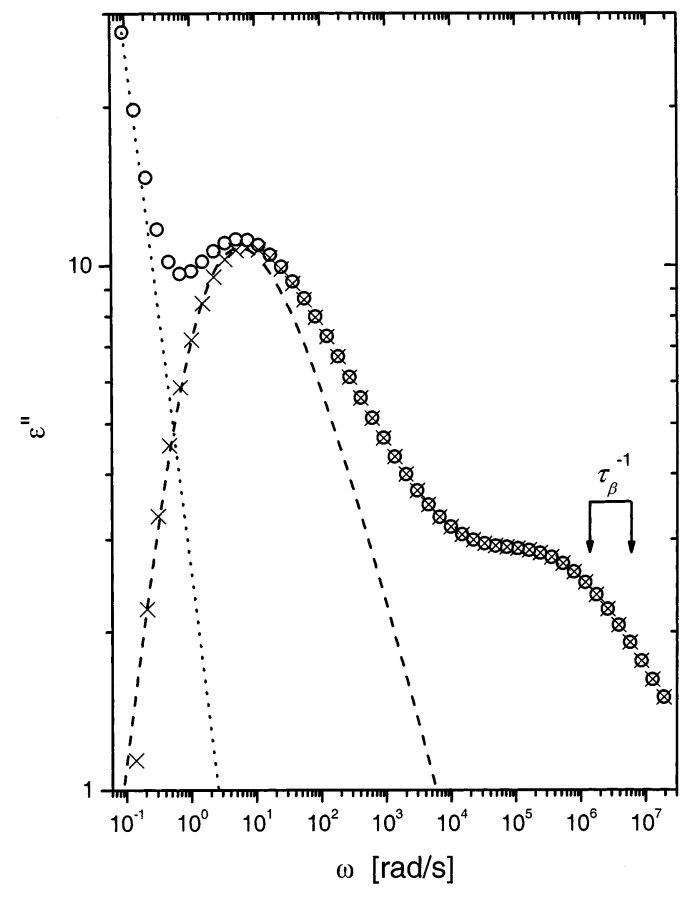

Figure 3. The dielectric loss for sorbitol at $286.4 \mathrm{~K}$ and $250 \mathrm{MPa}(\bigcirc)$ as measured and $(x)$ after subtraction of the dc-conductivity $\times$, the latter indicated by a dotted line. The dashed line is the fit of eq 5 to the low-frequency side of the spectrum. The double arrow denotes the range of $1 / \tau_{\beta}$ calculated from eq 4 for $\beta_{\mathrm{K}}=0.48 \pm 0.03$.

yields $\beta_{\mathrm{K}}=0.48 \pm 0.03$. From eq 4 , we then calculate that $\tau_{\beta}$ $=(4 \pm 4) \times 10^{-7} \mathrm{~s}$. This predicted position of the $\beta$-peak is consistent with the experimental spectrum, as shown in Figure 3.

We can compare the activation volume for the primary structural relaxation to the $\Delta V$ for two homologous polyalcohols, glycerol ${ }^{4,22}$ and xylitol. ${ }^{34}$ Because the activation volume depends on temperature $\left(\mathrm{d} V_{\mathrm{m}} / \mathrm{d} T \approx 0.055 \mathrm{~mL} \mathrm{~mol}^{-1} \mathrm{~K}^{-1}\right.$ for sorbitol) and often on pressure as well, we make the comparison at ambient pressure and temperatures at which the respective $\tau_{\alpha}$ $\approx 1 \mathrm{~s}$. Isotherms for the three liquids are shown in Figure 4. There is an increasing sensitivity to pressure with increasing molecular weight of the alcohol. The corresponding activation volumes are listed in Table 2, in which it can be seen that $\Delta V$ increases with increasing molecular size, although no simple relationship is evident.

Also shown in Table 2 is the dependence of the glass temperature on pressure. There is no significant trend in $\mathrm{d} T_{\mathrm{g}} /$ $\mathrm{d} P$ for the three materials. Given this constancy, we can make a prediction about the relative fragility of the polyalcohols. This fragility can be expressed as ${ }^{4}$

$$
m=\frac{\Delta V}{\ln 10 R \mathrm{~d} T_{\mathrm{g}} / \mathrm{d} P}
$$

Equation 6 indicates that the fragilities of the three polyalcohols will parallel the magnitude of their activation volumes. As seen in Table 2, this ordering is in agreement with the values of $m$ determined at ambient pressure. ${ }^{4,34}$ In fact, the ratio of $\Delta V$ over $m$ is nearly constant, $0.26 \pm 0.03$.

The dependence of the relaxation times on volume can be formally related to the ratio of the activation volume and isothermal compressibility, $\kappa_{\mathrm{T}},{ }^{35}$

$$
\left.\frac{\partial \tau_{\alpha}}{\partial V}\right|_{T}=(R T)^{-1} \frac{\Delta V}{\kappa_{\mathrm{T}}}
$$




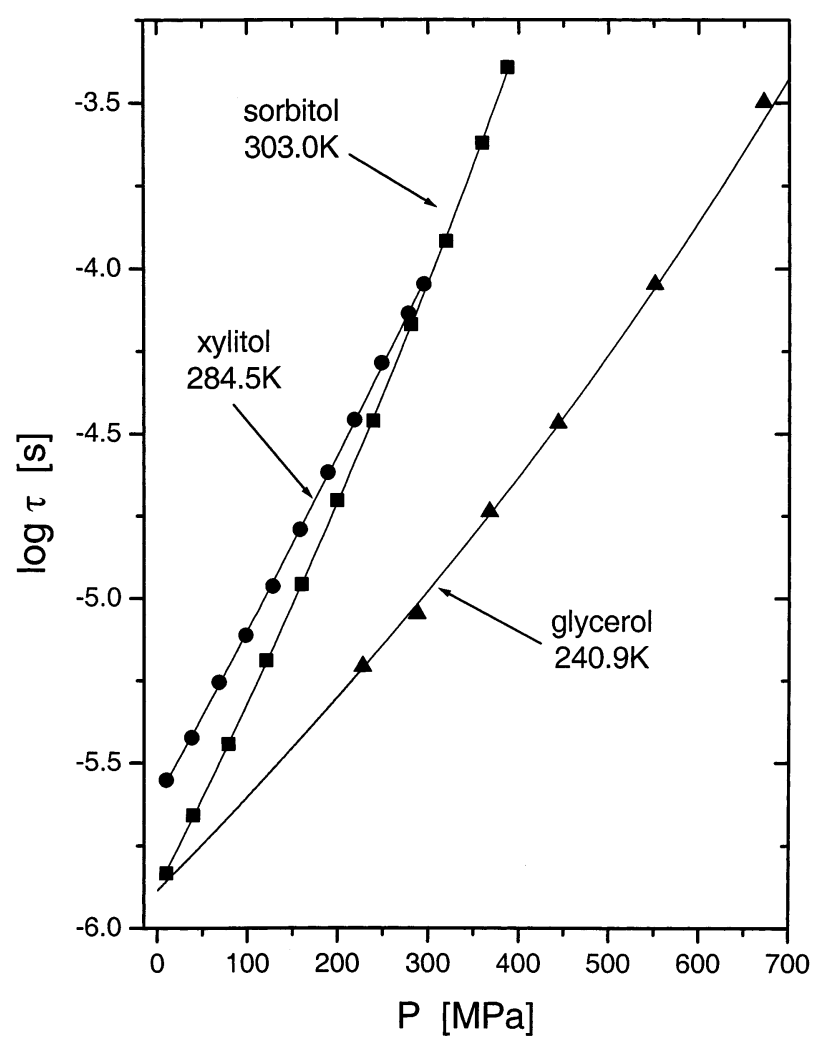

Figure 4. The structural relaxation times as a function of pressure for the three polyalcohols at respective temperatures for which $\tau_{\alpha}=1 \mathrm{~s}$ at ambient pressure.

Because $\kappa_{\mathrm{T}}$ invariably decreases with pressure (see eq 8 below), the volume dependence of $\tau_{\alpha}$ cannot be independent of pressure; that is, the relaxation times are not uniquely defined by the volume. The relative importance of temperature and density in governing the magnitude of the structural relaxation times can be quantified by calculating the ratio of the coefficients of isobaric, $\alpha_{P}\left(-\rho^{-1}(\partial \rho / \partial T)_{P}\right)$, and isochronal, $\alpha_{\tau}\left(-\rho^{-1}(\partial \rho / \partial T)_{\tau}\right)$, expansion. ${ }^{5}$ Previously, we have found that for nonassociated liquids, the absolute value of this ratio is on the order of unity, ${ }^{6-8}$ indicating that pressure and temperature exert similar effects on the structural relaxation times. However, for glycerol, a hydrogen-bonded glass former, Ferrer et al. ${ }^{5}$ obtained $-\alpha_{\tau} / \alpha_{P}$ $\approx 17$, implying a dominant effect of thermal energy on $\tau_{\alpha}$.

We calculate an isochronal expansion coefficient for sorbitol from the temperatures and pressures at which $\tau=1 \mathrm{~s}$, in combination with published $P V T$ data. The specific volume can be expressed using the Tait equation ${ }^{36}$

$$
v(T, P)=v_{0}\left[1-0.0894 \ln \left(1+\frac{P}{b_{0}+b_{1} T}\right)\right]
$$

Fitting the data of Naoki et al. ${ }^{37}$ for sorbitol, we obtain (in units of $\mathrm{g} / \mathrm{mL}$ ) $b_{0}=1134 \mathrm{MPa}$ and $b_{1}=-1.572 \mathrm{MPa} / \mathrm{K}$. The volume at ambient pressure, $v_{0}$, can be expressed as a polynomial in $T$

$$
\begin{array}{r}
v_{0}=0.6142+2.700 \times 10^{-4} T-2.0265 \times 10^{-7} T^{2}+ \\
5.483 \times 10^{-10} T^{3}
\end{array}
$$

Using the PVT results, we obtain from eq $8 \alpha_{P}=4.18 \times 10^{-4}$ $\mathrm{K}^{-1}$ for the isobaric expansivity at $P=0.1 \mathrm{MPa}$. Using eqs 7 and 8 , along with the $T_{\mathrm{g}}(P)$ determinations herein, we calculate $\alpha_{\tau}=-2.43 \times 10^{-3} \mathrm{~K}^{-1}$ for $\tau_{\alpha}=1 \mathrm{~s}$. We thus obtain for the ratio, $\left|\alpha_{\tau}\right| / \alpha_{P}=5.8$. This ratio is larger than unity, suggesting that temperature is the dominant variable governing the structural
TABLE 2: Comparison of Polyalcohols

\begin{tabular}{lcccccr}
\hline & \multicolumn{7}{c}{$\begin{array}{c}T_{\mathrm{g}}{ }^{a} \\
(\mathrm{~K})\end{array}$} & $\begin{array}{c}\mathrm{d} T_{\mathrm{g}} / \mathrm{d} P \\
(\mathrm{~K} / \mathrm{GPa})\end{array}$ & $\begin{array}{c}\Delta V^{b} \\
(\mathrm{ml} / \mathrm{mol})\end{array}$ & $m^{a}$ \\
\hline Sorbitol & $\mathrm{C}_{6} \mathrm{H}_{14} \mathrm{O}_{6}$ & 182.17 & 272 & $40 \pm 3$ & $33.3 \pm 0.3$ & 128 \\
xylitol & $\mathrm{C}_{5} \mathrm{H}_{12} \mathrm{O}_{5}$ & 152.1 & 253 & $34.3 \pm 0.1$ & $27.1 \pm 0.6$ & 94 \\
glycerol & $\mathrm{C}_{3} \mathrm{H}_{8} 0_{3}$ & 92.09 & 196 & $35 \pm 1$ & $13.3 \pm 1.1$ & 54 \\
\multicolumn{7}{c}{$\tau_{\alpha}=1 \mathrm{~s}$ at ambient pressure. ${ }^{b}$ At ambient pressure and the } \\
temperature at which $\tau=1 \mathrm{~s}$.
\end{tabular}

relaxation times of sorbitol. The value for sorbitol is smaller than that for glycerol, consistent with a reduced degree of H-bonding per molecule for sorbitol.

Note that the measurements herein are for $P>247 \mathrm{MPa}$, whereas the $P V T$ measurements of Naoki et al. ${ }^{37}$ were limited to $P<79 \mathrm{MPa}$. Thus, the calculation of the isochronal expansion coefficient requires significant extrapolation, resulting in a considerable uncertainty in the obtained $\left|\alpha_{\tau}\right| / \alpha_{P}$. We can corroborate this result using a relation proposed by Naoki et al. ${ }^{38}$ for the ratio of the activation energy at constant volume, $E_{V}$, to that at constant pressure, $E_{P}$

$$
E_{V} / E_{P}=1-\gamma(\partial T / \partial P)_{\tau}
$$

where $\gamma$ is the thermal pressure coefficient and the temperaturepressure coefficient at constant value of $\tau_{\alpha}=1 \mathrm{~s}$ is just the pressure coefficient of the glass temperature, $\mathrm{d} T_{\mathrm{g}} / \mathrm{d} P=0.040$ $\mathrm{K} / \mathrm{MPa}$. The magnitude of $E_{V} / E_{P}$ reflects directly the contribution of thermal energy to the temperature dependence of $\tau_{\alpha \cdot}{ }^{38}$ Using the Tait parameters (eq 8) above, we obtain $\gamma=3.31$ $\mathrm{MPa} / \mathrm{K}$ for sorbitol; whence $E_{V} / E_{P}=0.87$.

This result, as well as the large value of $\left|\alpha_{\tau}\right| / \alpha_{P}$, indicates that temperature, rather than density, governs the magnitude of the structural relaxation times. Although temperature variations cause changes in both the thermal energy and the density, for sorbitol, the latter has a much less consequential effect on $\tau_{\alpha}$. This agrees with the results of Ferrer et al. ${ }^{5}$ for glycerol, in which temperature was also found to be the dominant control variable. In contrast, for nonassociated liquids, both thermal energy and density are equally important for structural relaxation. ${ }^{6-9}$

\section{Summary}

Temperature is the governing variable in the structural relaxation of sorbitol. In this respect, sorbitol is similar to glycerol but distinct from nonassociated liquids, the relaxation times of which depend strongly on both density and thermal energy. It is ironic that the role of the temperature should be paramount in $\mathrm{H}$-bonded liquids, because it is these liquids that exhibit fragilities ${ }^{4}$ and $\tau_{\beta}$ that change with pressure. This apparent contradiction may provoke efforts to better understand the relationship between the chemical structure of glass-formers and their temperature and pressure dependences.

The fact that density, or volume, is not the dominant control variable for sorbitol calls into question the concept of an activation volume for this material. Because structural relaxation in sorbitol and glycerol is an activated process, the activation volume should be regarded as the difference in molar volume between the activated and initial species, ${ }^{21}$ changes in interstitial volume effected by pressure altering the potential barriers. The relatively weak effect of pressure is due to two competing effects. Compression promotes intermolecular constraints and thus inhibits relaxation, but pressure also reduces the extent of hydrogen bonding, ${ }^{11,39,40}$ which enhances local mobility. 
The pressure coefficient of the glass temperature for sorbitol is equal to $\mathrm{d} T_{\mathrm{g}} / \mathrm{d} P$ for glycerol and to that for xylitol as well. However, the activation volumes of these polyalcohols are all different, $\Delta V$ increasing with molecular size. From the relationship of $\Delta V$ and $\mathrm{d} T_{\mathrm{g}} / \mathrm{d} P$ to fragility (eq 6), this leads to the prediction that the latter increases as sorbitol $>$ xylitol $>$ glycerol. The same ordering is obtained from direct measurements of $m$ at ambient pressure for the three glass-formers.

The secondary relaxation in sorbitol exhibits a weak dependence on pressure, $\Delta V$ being an order of magnitude smaller than that for structural relaxation. A sensitivity of $\tau_{\beta}$ to pressure implies that the $\beta$-relaxation is intermolecular in nature. Consistent with this result, the approximate magnitude of $\tau_{\beta}$ is in accord with eq 4, which assumes the Johari-Goldstein secondary process to be the precursor to a fully intermolecularly cooperative structural relaxation.

Acknowledgment. The Office of Naval Research and the Committee for Scientific Research, Poland (KBN, Grant No. 2PO3B 033 23), are gratefully acknowledged for financial support. We thank K. L. Ngai and R. Casalini for stimulating discussions and $\mathrm{H}$. Wagner for providing the ambient pressure data for sorbitol.

\section{References and Notes}

(1) Paluch, M.; Pawlus, S.; Roland, C. M. Macromolecules 2002, 35, 7338. 10932.

2) Paluch, M.; Roland, C. M.; Pawlus, S. J. Chem. Phys. 2002, 116,

(3) Williams, G. Trans. Faraday Soc. 1966, 62, 2091.

(4) Paluch, M.; Casalini, R.; Hensel-Bielowka, S.; Roland, C. M. J. Chem. Phys. 2002, 116, 9839.

(5) Ferrer, M. L.; Lavrence, Ch.; Demirjian, B. G.; Kivelson, D.; AlbaSimonesco, Ch.; Tarjus, G. J. Chem. Phys. 1998, 109, 8010.

(6) Paluch, M.; Roland, C. M.; Casalini, R.; Meier, G.; Patkowski, A. J. Chem. Phys., submitted for publication.

(7) Paluch, M.; Casalini, R.; Roland, C. M. Phys. Rev. B 2002, 66, 092202 . 1188 .

(8) Paluch, M.; Roland, C. M.; Best, A. J. Chem. Phys. 2002, 117,

(9) Paluch, M.; Roland, C. M.; Gapinski, J.; Patkowski, A. J. Chem. Phys., submitted for publication.
(10) Casalini, R.; Paluch, M.; Roland, C. M. J. Phys. Chem. B, submitted. (11) Naoki, M.; Katahira, S. J. Phys. Chem. 1991, 95, 431.

(12) Nozaki, R.; Suzuki, D.; Ozawa, S.; Shiozaki, Y. J. Non-Cryst. Solids 1998, 235-237, 393.

(13) Olsen, N. B. J. Non-Cryst. Solids 1998, 235-237, 399.

(14) Wagner, H.; Richert, R. J. Non-Cryst. Solids 1998, 242, 19.

(15) Wagner, H.; Richert, R. J. Phys. Chem. B 1999, 103, 4071.

(16) Faivre, A.; Niquet, G.; Maglione, M.; Fornazero, J.; Jal, J. F.; David,

L. Eur. Phys. J. B 1999, 10, 277.

(17) Fujima, T.; Frusawa, H.; Ito, K.; Hayakawa, R. Jpn. J. Appl. Phys. 2000, 39, 744 .

(18) Richert, R. Europhys. Lett. 2001, 54, 767.

(19) Döss, A.; Paluch, M.; Sillescu, H.; Hinze, G. Phys. Rev. Lett. 2002, 88,095701 .

(20) Simatos, D.; Blond, G.; Roudaut, G.; Champion, D.; Perez, J.; Faivre, A. L. J. Therm. Anal. 1996, 47, 1419.

(21) Glasstone, S.; Laidler, K. J.; Eyring, H. Theory of Rate Processes; McGraw-Hill, New York, 1941.

(22) Johari, G. P.; Whalley, E. Faraday Symp. Chem. Soc. 1972, 6, 23.

(23) Paluch, M.; Ziolo, J.; Rzoska, S. J.; Habdas, P.; Ziolo, J. J. Phys.: Condens. Matter 1996, 8, 10885.

(24) Goldstein, M. J. Chem. Phys. 1969, 51, 3728.

(25) Johari, G. P. J. Chem. Phys. 1973, 58, 1766.

(26) Johari, G. P.; Goldstein, M. J. Chem. Phys. 1970, 53, 2372.

(27) Meier, G.; Gerharz, B.; Boese, D.; Fischer, E. W. J. Chem. Phys. 1991, 94, 3050 .

(28) Hensel-Bielowka, S.; Ziolo, J.; Paluch, M.; Roland, C. M. J. Chem. Phys. 2002, 117, 2317.

(29) Köplinger, J.; Kasper, G.; Hunklinger, S. J. Chem. Phys. 2000, 113 4701 .

(30) Ngai, K. L. Phys. Rev. E 1998, 57, 7346.

(31) Leon, C.; Ngai, K. L.; Roland, C. M. J. Chem. Phys. 1999, 110, 11585 .

(32) Kohlrausch, R. Ann. Phys. Chem. 1847, 12, 393.

(33) Williams, G.; Watts, D. C. Trans. Faraday Soc. 1970, 66, 80.

(34) Paluch, M.; Roland, C. M.; see also reference 4.

(35) Forsman, H.; Andersson, P.; Backstrom, G. J. Chem. Soc., Faraday Trans. 1986, 82, 857 .

(36) Yi, Y. X.; Zoller, P. J. Polym. Sci., Polym. Phys. Ed. 1993, 31, 779.

(37) Naoki, M.; Ujita, K.; Kashima, S. J. Phys. Chem. 1993, 97, 12356. (38) Naoki, M.; Endou, H.; Matsumoto, K. J. Phys. Chem. 1987, 91, 4169 .

(39) Poole, P. H.; Sciortino, F.; Grande, T.; Stanley, H. E.; Angell, C. A. Phys. Rev. Lett. 1994, 73, 1632.

(40) Cook, R. E.; King, H. E.; Peiffer, D. G. Phys. Rev. Lett. 1992, 69, 30732 . 